\title{
ON THE WEAK FINITE STOCHASTIC REALIZATION PROBLEM
}

\author{
G. Picci \\ CNR-LADSEB and \\ Istituto di Elettrotechnica \\ Università di Padova \\ Italy
}

J.H. van Schuppen Mathematical Centre

Amsterdam

The Netherlands

\section{ABSTRACT}

The weak finite stochastic realization problem is given a stationary finite valued stochastic process to show existence of and to classify all minimal finite stochastic systems whose output equals the given process in distribution. In this paper the characterization of minimal realizations is investigated and reduced to a factorization problem for positive matrices. The latter problem is discussed and solved in a rather special case.

\section{INTRODUCTION}

The purpose of this paper is to present a problem formulation of the weak finite stochastic realization problem and to indicate the major current question for. this problem: the positive factorization problem.

The weak finite stochastic realization problem is given a finite valued stationary stochastic process to show existence of and to classify all minimal finite stochastic systems whose output equals the given process in distribution. In contrast with this, the strong finite stochastic realization problem is to answer the same question but under the condition that the output process equals the given process almost surely.

The motivation of this problem is the area of control and prediction for systems with point process observations. Examples of practical problems in this area are the control of queues, the prediction of traffic intensities, the estimation of software reliability, and the estimation of certain biomedical signals. These practical problems may be modelled by finite stochastic systems. The prediction and control problems for this class of systems, under the assumption that the parameter values are known, have been considered. Practical application of these results demands the solution of the system identification problem and the stochastic realization problem for finite stochastic systems.

A brief description of the content of the paper follows. A problem formulation and a definition of a finite stochastic system is given in section 2 . The characterization of minimal realizations, and the problem of positive factorization is discussed in section 3 . 


\section{PROBLEM FORMULATION}

Below a definition is given of a finite stochastic system and the weak finite stochastic realization problem is formulated.

Let $(\Omega, F, P)$ be a complete probability space and $T=Z$ be the time index set. The conditional independence relation for a triple of $\sigma$-algebra's $F_{1}, F_{2}, G$ is defined to satisfy

$$
E\left[x_{1} x_{2} \mid G\right]=E\left[x_{1} \mid G\right] E\left[x_{2} \mid G\right] \text { a.s. }
$$

for all $x_{1} \in L^{+}\left(F_{1}\right), x_{2} \in L^{+}\left(F_{2}\right)$, notation $\left(F_{1} G, F_{2}\right) \in C I$.

Here $L^{+}\left(F_{1}\right)$ is the set of all positive $F_{1}$ measurable random variables. The smallest $\sigma$-algebra with respect to which a random variable $\mathrm{x}$ is measurable is denoted $\mathrm{F}^{\mathrm{X}}$, and that containing the $\sigma$-algebra's $G, H$ by $G \vee H$.

2.1. DEFINITION. A finite stochastic system is a collection

$\sigma=\left\{\Omega, \mathrm{F}, \mathrm{P}, \mathrm{T}, \mathrm{Y}, \mathrm{B}_{\mathrm{y}}, \mathrm{X}, \mathrm{B}_{\mathrm{X}}, \mathrm{y}, \mathrm{X}\right\}$ where $\{\Omega, \mathrm{F}, \mathrm{P}\}$ is a complete probability space, $T=Z, Y, X$ are finite sets called respectively the output space and the state space, $B_{y}, B_{x}$ are the $\sigma$-algebra's on $Y, X$ generated by all subsets of $\mathrm{Y}, \mathrm{X}, \mathrm{y}: \Omega \times \mathrm{T} \rightarrow \mathrm{Y}, \mathrm{X}: \Omega \times \mathrm{T} \rightarrow \mathrm{X}$ are stochastic processes called respectively the output process and the state process, such that for all $t \in T$

$$
\left(F_{t}^{y^{+}} \vee F_{t}^{x^{+}}, F^{X t}, F_{t}^{x^{-}} \vee F_{t-1}^{y^{-}}\right) \in C I,
$$

where $\mathrm{F}_{\mathrm{t}}^{\mathrm{y}^{+}}=\sigma\left(\left\{\mathrm{y}_{\mathrm{s}}, \forall s \geq \mathrm{t}\right\}\right), \mathrm{F}_{\mathrm{t}}^{\mathrm{x}^{-}}=\sigma\left(\left\{\mathrm{x}_{\mathrm{s}}, \forall s \leq \mathrm{t}\right\}\right)$.

Notation: $\sigma \in$ FSL.

In a stochastic system one exhibits, besides the externally available output process, the underlying state process. The above defined system is called finite because $Y, X$ are finite sets. The definition stated above has been first given in [7]. In the stochastic automata literature a finite stochastic system is called a stochastic automaton of Mealey-type [6].

2.2. PROBLEM. The weak finite stochastic realization problem (WFSRP) is given a stationary stochastic process on $T=2$, with values in a finite set $Y$, and the class of finite stochastic systems, to solve the following subproblems: a. does there exist a finite stochastic system

$$
\sigma=\left\{\Omega, F, P, T, Y, B_{y}, X, B_{x}, y, x\right\} \in F S \Sigma
$$

such that the output process y equals the given process in distribution?; if such a system exists, it is called a weak finite stochastic realization of the given process;

b. classify all minimal weak finite stochastic realizations, where minimal refers to the number of elements in the state space; this involves: 
1. the characterization of minimal realizations;

2. the classification as such;

3. the relation between minimal realizations;

4. the construction of an algorithm that constructs all minimal realizations.

The above problem has first been posed by D. Blackwell and L. Koopmans [2], although in a somewhat different form. During the $1960^{\prime}$ s several contributions to the problem have been given, see the book by A. Paz [6] for references. However, little progress has been made on the problem.

The existence question of the weak finite stochastic realization problem has been solved. The solution is due to A. Heller [4]; see [7] for an alternative proof. However, there is no algorithm that allows one to construct a stochastic realization.

Of the classification subproblem of 2.2 little is known. Let's consider the first question: what are necessary and sufficient conditions for a weak finite stochastic realization to be minimal? This question is unsolved and difficult. To discuss it in more detail attention is restricted to the static problem; this is done in section 3 .

\section{THE POSITIVE FACTORIZATION PROBLEM}

A major question in the weak finite stochastic realization problem is the characterization of minimal realizations. In this section a restricted case of this question is discussed, namely that in which there is only a past and a present and one is asked to construct a state. To be precise, one has the following problem.

3.1. PROBLEM. Assume given two finite sets $\mathrm{Y}^{+}, \mathrm{Y}^{-}$, and a frequency function $\mathrm{P}_{0}: \mathrm{Y}^{+} \times \mathrm{Y}^{-} \rightarrow R_{+}$(i.e. $\sum_{i \in \mathrm{Y}^{+}, j \in \mathrm{Y}^{-}} \mathrm{P}_{0}(i, j)=1$ ).

a. Does there exist a collection $\sigma=\left\{Y^{+}, Y^{-}, X, P\right\}$ such that 1. $\sigma=\left\{Y^{+}, Y^{-}, X, P\right\} \in F P E$, where $X$ is a finite set, $\mathrm{p}: \mathrm{Y}^{+} \times \mathrm{X} \times \mathrm{Y}^{-} \rightarrow \mathrm{R}_{+}$is a frequency function, and with respect to the canonical variables one has $\left(\mathrm{F}^{+}, \mathrm{F}^{\mathrm{x}}, \mathrm{F}^{-}\right) \in \mathrm{CI}$;

2. the restriction of $\mathrm{p}$ to $\mathrm{Y}^{+} \times \mathrm{Y}^{-}$equals $\mathrm{p}_{0}$. Then $\sigma$ is called a probabilistic realization of $\left\{\mathrm{Y}^{+}, \mathrm{Y}^{-}, \mathrm{P}_{0}\right\}$.

b. Classify all minimal probabilistic realizations of $\left\{\mathrm{Y}^{+}, \mathrm{Y}^{-}, \mathrm{P}_{0}\right\}$. A probabilistic realization is called minimal if $X$ has the smallest number of elements of all probabilistic realizations.

The existence problem in 3.1 is trivial. The major question is the characterization of minimal realization and their classification. 
An equivalent condition for a probabilistic realization is needed and will be given below. First some notation is introduced. The set of the integers is denoted by $z$, and for $n \in Z_{+}, Z_{n}=\{1,2,3, \ldots, n\}$. Furthermore, for $k \in Z_{+}, R_{n}^{k}$ denotes the vector space with components in $R_{+}$, and $R_{+}^{k \times m}$ the set of positive matrices. For $k \in z_{+}$, with $e_{k}^{T}=\left(\begin{array}{llll}1 & 1\end{array} \ldots\right.$ 1 $) \in R_{+}^{k}$,

$$
\mathrm{S}^{\mathrm{k}}=\left\{\mathrm{x} \in \mathrm{R}_{+}^{\mathrm{k}} \mid \mathrm{e}_{\mathrm{k}}^{\mathrm{T}} \mathrm{x}=1\right\}
$$

will be called the set of stochastic vectors. The set of stochastic matrices, column wise, is defined by

$$
S^{k \times m}=\left\{Q \in R_{+}^{k \times m} \mid e_{k}^{T} Q=e_{m}^{T}\right\} .
$$

Given a finite set $\mathrm{Y}, \#(\mathrm{Y})$ denotes the number of elements in $\mathrm{Y}$. If $\mathrm{Y}^{+}, \mathrm{Y}^{-}$are finite sets, $k=\#\left(Y^{-}\right), p: Y^{+} \times Y^{-} \rightarrow R_{+}$is a frequency function, then define the conditional probability matrix $Q_{y}+y^{-} \epsilon S^{k \times m}$ by

$$
Q_{y^{+} / y^{-}}^{i j}=p(i, j) / p(j)
$$

if this is well defined, and zero otherwise.

3.2. PROPOSITION. Assume given two finite sets $\mathrm{Y}^{+}, \mathrm{Y}^{-}$with $\#\left(\mathrm{Y}^{+}\right)=\mathrm{k}, \#\left(\mathrm{Y}^{-}\right)=\mathrm{m}$, and a frequency function $\mathrm{P}_{0}: \mathrm{Y}^{+} \times \mathrm{Y}^{-} \rightarrow \mathrm{R}_{+}$. The following statements are then equivalent:

a. there exists a probabilistic realization $\left\{Y^{+}, Y^{-}, X, p\right\} \in \operatorname{FP} \Sigma$ with $\#(X)=n$;

b. there exist $n \in Z_{+}, Q_{1} \in s^{k \times n}, Q_{2} \in s^{n \times m}$ such that $Q_{y}+y^{-}=Q_{1} \cdot Q_{2}$;

c. there exist $n \in Z_{+}, \pi \in S^{n}$, and for all $i \in Z_{n}$ there exist $p_{i} \in S^{k}$, $r_{i} \in S^{m}$, such that

$$
p_{0}(i, j)=\sum_{s=1}^{n} \pi_{s} p_{s}^{i} r_{s}^{j T}
$$

Proof. The elementary proof is omitted.

The characterization 3.2.b., of a factorization of a stochastic matrix into the product of two stochastic matrices, will be used in the sequel. The proof of 3.2 shows that then $Q_{1}=Q_{y}+x, Q_{2}=Q_{x} \mid y^{-}$.

In the following attention will be restricted to the factorization of positive matrices. It is easily proven that if one has a factorization of a stochastic matrix in a product of two positive matrices, then one can modify the factorization into one with two stochastic matrices.

3.3. DEFINITION. Given $Q \in \mathrm{R}_{+}^{\mathrm{k} \times \mathrm{m}}$.

a. A positive factorization of $Q$ is a factorization of the form

$$
\mathrm{Q}=\mathrm{A} \cdot \mathrm{B}
$$

where $A \in R_{+}^{k \times n}, B \in R_{+}^{n \times m}$ for some $n \in Z_{+}$; 
b. A minimal positive factorization of $Q$ is

1. a positive factorization of $Q$, say

$Q=A \cdot B$, with $A \in R_{+}^{k \times n}, B \in R_{+}^{n \times m}$;

2. if $Q=C \cdot D, C \in R_{+}^{k \times n_{1}}, D \in R_{+}^{n_{1} \times m}$,

is any other positive factorization of $Q$, then $n_{1} \geq n$.

One then calls $n$ the positive rank of $Q$, notation $n=p o s-\operatorname{rank}(Q)$.

3.4. PROBLEM. The minimal positive factorization problem is, given $Q \in \mathrm{R}_{+}^{\mathrm{k} \times \mathrm{m}}$,

a. to give necessary and sufficient conditions for a positive factorization of $Q$ to be minimal;

b. to classify all minimal positive factorizations of $Q$;

c. to construct an algorithm that produces all minimal positive factorizations of $Q$.

\subsection{REMARKS.}

1. The minimal positive factorization problem is seen to be the restriction of the characterization of minimal weak finite stochastic realizations. This problem is therefore of interest.

2. The problem is also of interest to the area of positive systems, see [5, Chap.6]. The realization problem for positive systems reduces in the static case to the minimal positive factorization problem.

3. In the literature on linear algebra, see [1,3] for some references, the above defined problem is not mentioned. However it is known that the positive rank of a matrix differs in general from the linear rank. In the literature the problem had been posed to give necessary and sufficient conditions for these two rank concepts to be the same. In our opinion this question is uninteresting, the interesting problem being the minimal positive factorization problem.

4. A geometric interpretation of a positive factorization can be given as follows. One has a positive factorization $Q=A$. B iff $C_{1}$ is contained in $\mathrm{C}_{2}$, where

$$
\begin{aligned}
& \left.\mathrm{C}_{1}=\text { conv. (columns of } \mathrm{Q}\right) \subset \mathrm{R}_{+}^{\mathrm{k}} \\
& \mathrm{C}_{2}=\text { conv. (columns of } \mathrm{A} \text { ), }
\end{aligned}
$$

and the right hand side denotes the convex hull generated by the denoted set. Further a positive factorization is minimal iff $\mathrm{C}_{2}$ is spanned by as few vertices as possible. The minimal positive factorization problem may then be interpreted as the search for a polyhedral cone that contains $c_{l}$, lies in $R_{+}^{k}$ and has as few vertices as possible.

Let $Q \in R_{+}^{k \times k}$. For $k=1,2$, and 3 it is easily shown that the positive rank of $Q$ equals the linear rank of $Q$. However this is not true for $k=4$. 
3.6. Examp 1e. Let

$$
Q=\left(\begin{array}{llll}
1 & 0 & 0 & 1 \\
1 & 1 & 0 & 0 \\
0 & 1 & 1 & 0 \\
0 & 0 & 1 & 1
\end{array}\right) \in \mathrm{R}_{+}^{4 \times 4}
$$

Then 1 in $-\operatorname{rank}(Q)=3<4=\operatorname{pos}-\operatorname{rank}(Q)$.

That in example 3.6 the positive rank of $Q$ is indeed four follows from the following result.

3.7. PROPOSITION. Let $Q \in \mathrm{R}_{+}^{\mathrm{k} \times \mathrm{k}}$, and denote the columns of $Q$ by $q_{i}$, $i \in z_{k}$. Assume that

1. $\mathrm{k} \geq 4$;

2. $\left\{q_{i}\right.$, $\left.i \in z_{k}\right\}$ lie on different faces of $R_{+}^{k \times k}$.

Then pos $-\operatorname{rank}(Q)=k$.

The proof is deferred to a future publication. The investigation of the minimal positive factorization problem is being continued.

\section{CONCLUDING REMARKS}

In this paper the weak finite stochastic realization has been posed. The current open problem is the characterization of minimal realizations. Through reduction it has been shown that this problem is equivalent to the minimal positive factorization problem. The latter problem is currently under investigation.

At the meeting at which this paper has been presented the strong finite stochastic realization problem has also been discussed; see [3] for an exposition.

\section{REFERENCES}

1. A. Berman, R.J. Plemmons, Nonnegative matrices in the mathematical sciences, Academic Press, New York, 1979.

2. D. Blackwe11, L. Koopmans, On the identifiability problem for functions of finite Markov chains, Ann. Math. Statist., 28(1957)pp.1011-1015.

3. S.L. Campbe1l, G.D. Poole, Computing nonnegative rank factorizations, Linear Algebra and its App1.35(1981),pp. 175-182.

4. A. Heller, On stochastic processes derived from Markov chains, Ann. Math. Statist., 36(1965), pp.1286-1291.

5. D.G. Luenberger, Introduction to dynamic systems - Theory, models, and applications, Wiley, New York, 1979.

6. A. Paz, Introduction to probabilistic automata, Academic Press, New York, 1971.

7. G. Picci, On the internal structure of finite-state stochastic processes, in "Recent Developments in Variable Structure Systems, Economics, and Biology", Proc. of a U.S.-Italy Seminar, Taormina, Sicily, 1977, Lecture Notes in Econ. and Mathematical Systems, volume 162, Springer-Verlag, Berlin, 1978.

8. J.H. van Schuppen, The strong finite stochastic realization problem - Preliminary results, in "Analysis and Optimization of Systems", A. Bensoussan, J.L. Lions eds., Lecture Notes in Control and Info. Sci., volume 44, SpringerVerlag, Berlin, 1982, pp. 179-190. 